\title{
Parahydrogen-enhanced zero-field nuclear magnetic resonance
}

\author{
T. Theis ${ }^{1,2}$, P. Ganssle ${ }^{1,2}$, G. Kervern ${ }^{1,2}$, S. Knappe ${ }^{3}$, J. Kitching ${ }^{3}$, M. P. Ledbetter ${ }^{4}$, D. Budker ${ }^{4,5}$ \\ and A. Pines ${ }^{1,2 \star}$
}

Nuclear magnetic resonance, conventionally detected in magnetic fields of several tesla, is a powerful analytical tool for the determination of molecular identity, structure and function. With the advent of prepolarization methods and detection schemes using atomic magnetometers or superconducting quantum interference devices, interest in NMR in fields comparable to the Earth's magnetic field and below (down to zero field) has been revived. Despite the use of superconducting quantum interference devices or atomic magnetometers, low-field NMR typically suffers from low sensitivity compared with conventional high-field NMR. Here we demonstrate direct detection of zero-field NMR signals generated through parahydrogen-induced polarization, enabling high-resolution NMR without the use of any magnets. The sensitivity is sufficient to observe spectra exhibiting ${ }^{13} \mathrm{C}-{ }^{1} \mathrm{H}$ scalar nuclear spin-spin couplings (known as $\mathrm{J}$ couplings) in compounds with ${ }^{13} \mathrm{C}$ in natural abundance, without the need for signal averaging. The resulting spectra show distinct features that aid chemical fingerprinting.

N uclear magnetic resonance (refs 1,2 ) in low or zero magnetic field has long been viewed as a curiosity owing to the low nuclear spin polarization, the poor sensitivity of inductive pickup coils at low frequencies and the absence of site-specific chemical shifts. Here, we show that the first two objections can be removed and we obtain high-resolution, high-signal-to-noise-ratio, zero-field NMR spectra that are rich in information. This facilitates the development of portable sensors for chemical analysis and imaging by elimination of cryogenically cooled superconducting magnets. Additionally, working in low or zero magnetic field yields narrow lines and accurate determination of line positions, owing to the high absolute field homogeneity and stability. These features have enabled chemical analysis through ${ }^{129} \mathrm{Xe}$ chemical shifts ${ }^{3}$ and spin-spin or $J$ couplings in ${ }^{1} \mathrm{H}-{ }^{13} \mathrm{C},{ }^{1} \mathrm{H}-{ }^{29} \mathrm{Si}$ and ${ }^{1} \mathrm{H}-{ }^{19} \mathrm{~F}$ in low or zero magnetic field ${ }^{4-6}$. Atomic magnetometers ${ }^{7,8}$ and superconducting quantum interference devices ${ }^{9}$ are sensitive to low-frequency signals, offering dramatically improved signal-tonoise ratio (compared with inductive pickup coils) in low-field NMR (refs 4,10,11) and magnetic resonance imaging ${ }^{12,13}$.

Despite the use of atomic magnetometers or superconducting quantum interference devices, low-field NMR using samples thermally prepolarized in a permanent magnet typically suffers from low signal-to-noise ratio compared with inductively detected high-field NMR, in part because of the low polarization available from thermalization in a permanent magnet. To avoid this difficulty, in this work we produce large nuclear-spin polarization in zero-field NMR by employing the technique of parahydrogeninduced polarization (PHIP), whereby order from the singlet state of parahydrogen is transferred to a molecule of interest, either by hydrogenation ${ }^{14-17}$ or through reversible chemical exchange ${ }^{18,19}$. By flowing molecular hydrogen through an iron oxide catalyst at sufficiently low temperature (see Methods), it is possible to realize nearly $100 \%$ conversion of orthohydrogen to parahydrogen.
This results in significant signal enhancements compared with that obtained using thermal polarization, which is typically in the range of $10^{-5}-10^{-6}$. Before proceeding, it is worth noting that light-induced drift can also be used for enrichment of nuclear-spin isomers in other molecules ${ }^{20,21}$, although the demonstrated enrichments are significantly lower, of the order of $2 \%$. When combined with sensitive atomic magnetometers for detection of nuclear spin magnetization, PHIP enables NMR without any magnets. The sensitivity is sufficient to easily observe complex spectra exhibiting ${ }^{1} \mathrm{H}-{ }^{13} \mathrm{C} \mathrm{J}$ couplings in compounds with ${ }^{13} \mathrm{C}$ in natural abundance in just a few transients, a task that would require considerable signal averaging using thermal prepolarization. Although PHIP has been investigated in a variety of magnetic fields, ranging from the Earth's field to high field, observation of the resulting NMR signals has always been carried out in finite magnetic field. To the best of our knowledge, the work reported here represents the first direct observation of PHIP in a zero-field environment. We show that polarization can be transferred through a number of chemical bonds to remote parts of a molecule, and that zero-field spectroscopy can be used to distinguish between different isotopomers in ethylbenzene, the product of hydrogenation of styrene. The mechanism by which observable magnetization is generated from the parahydrogenderived singlet order requires only the presence of a heteronucleus, similar to the work of ref. 22, in contrast to a more commonly observed mechanism relevant to high field, which requires chemical-shift differences at the sites of the parahydrogen-derived protons. Furthermore, our results are of particular interest in the context of recent work demonstrating that the lifetime of singlet polarization in low fields can considerably exceed the relaxation time $T_{1}$ of longitudinal magnetization ${ }^{23,24}$. These demonstrations of increased singlet lifetime relied on field cycling and high-field inductive detection, and our methodology may provide for more direct observation and exploitation of these effects.

\footnotetext{
${ }^{1}$ Materials Science Division, Lawrence Berkeley National Laboratory, Berkeley, California 94720, USA, ${ }^{2}$ Department of Chemistry, University of California at Berkeley, Berkeley, California 94720-3220, USA, ${ }^{3}$ Time and Frequency Division, National Institute of Standards and Technology, 325 Broadway, Boulder, Colorado 80305, USA, ${ }^{2}$ Department of Physics, University of California at Berkeley, Berkeley, California 94720-7300, USA, ${ }^{5}$ Nuclear Science Division, Lawrence Berkeley National Laboratory, Berkeley, California 94720, USA. *e-mail: pines@berkeley.edu.
} 


\section{Zero-field NMR}

Zero-field NMR spectroscopy of samples magnetized by thermal prepolarization in a permanent magnet was discussed in refs 4,25 . In an isotropic liquid at zero magnetic field, the only terms in the NMR Hamiltonian are the spin-spin $J$ couplings, $H_{J}=\sum \hbar J_{j k} \mathbf{I}_{j} \cdot \mathbf{I}_{k}$. In the important case of $\mathrm{AX}_{N}$ systems, where both $\mathrm{A}$ and $\mathrm{X}$ are spin-1/2 particles, and each $\mathrm{X}$ spin couples to A with the same strength $J$, the resulting zero-field $J$ spectra are simple and straightforward to interpret, consisting of a single line at $J$ for $\mathrm{AX}$, a single line at $3 J / 2$ for $\mathrm{AX}_{2}$ and two lines, one at $J$ and one at $2 J$, for $\mathrm{AX}_{3}$. For larger molecules, as employed in the present work, long-range couplings to extra spins lead to splitting of these lines; however, the overall positions of the resulting multiplets remain unchanged. At present we rely on numerical spin simulations (presented in detail in Supplementary Information) to understand the splitting pattern; however, we anticipate that an approach based on perturbation theory will probably yield simple rules for interpretation of the zero-field splitting pattern.

Zero-field spectroscopy using parahydrogen-induced polarization differs from the case of thermal polarization in both the initial density matrix and in the method of excitation. In the case of homogeneous catalysis, the product molecule starts out with two parahydrogen-derived spins in a singlet state. Averaging over random hydrogenation events and subsequent evolution under the $J$-coupling Hamiltonian lead to an equilibrium density matrix described by pairs of heteronuclear and homonuclear scalar spin pairs, $\rho_{0}=\sum a_{j k} \mathbf{I}_{j} \cdot \mathbf{I}_{k}$, which bears no magnetic moment, and is static under the $J$-coupling Hamiltonian. Observable magnetization oscillating along the $z$ direction, to which the magnetometer is sensitive, can be produced by applying a pulse of d.c. magnetic field $B$ in the $z$ direction. Immediately following such a pulse, the density matrix contains terms of the form $\sin \eta\left(I_{j x} I_{k y}-I_{j y} I_{k x}\right)$, where $\eta=B t_{\mathrm{p}}\left(\gamma_{j}-\gamma_{k}\right), t_{\mathrm{p}}$ is the pulse duration and $\gamma_{j}$ is the gyromagnetic ratio of spin $j$. Subsequent evolution under the $J$-coupling Hamiltonian results in terms in the density matrix of the form $\left(I_{j, z}-I_{k, z}\right) \sin \eta \sin \left(J_{j k} t\right)$, which produces magnetization oscillating in the $z$ direction. The dependence on $\eta$ highlights the role of a heteronucleus in the symmetry breaking of the parahydrogenderived scalar order. Numerical spin simulations of the propagation of the parahydrogen-derived scalar order through the molecule and the dependence of the coherence amplitude on pulse area $\eta$ for a heteronuclear spin pair with scalar order are presented in Supplementary Information. A more detailed analysis of the polarization transfer will be presented in a forthcoming publication.

\section{Spectroscopy with zero-field PHIP}

The zero-field spectrometer used in this work is similar to that of ref. 4 and is shown in Fig. 1a. The noise spectrum of the magnetometer is shown in Fig. 1b, and the pulse sequence is shown in Fig. 1c. We carried out zero-field PHIP spectroscopy in hydrogenation reactions of styrene (which forms ethylbenzene), 3-hexyne (hexene and hexane), 1-phenyl-1-propyne (1-phenyl-1propene) and dimethylacetylenedicarboxlyate (dimethylmaleate). In measurements presented in the main text, parahydrogen was bubbled through the solution for $\sim 10 \mathrm{~s}$, the flow was halted and excitation pulses of d.c. magnetic field were applied in the $z$ direction with $\eta=\pi / 2$ for ${ }^{13} \mathrm{C}$ and protons. The resulting $z$ magnetization was recorded by the atomic magnetometer. The rate of hydrogenation can be monitored by the signal amplitude as a function of time, as presented in Supplementary Information. More details of the experimental set-up and procedures can be found in Methods.

Single shot, zero-field PHIP spectra of ethylbenzene- $\beta^{13} \mathrm{C}$ (labelled ${ }^{13} \mathrm{CH}_{3}$ group), and ethylbenzene- $\alpha{ }^{13} \mathrm{C}$ (labelled ${ }^{13} \mathrm{CH}_{2}$ group), synthesized from labelled styrene, are shown in black in Fig. 2a,b, respectively. The ethylbenzene molecule is shown in the
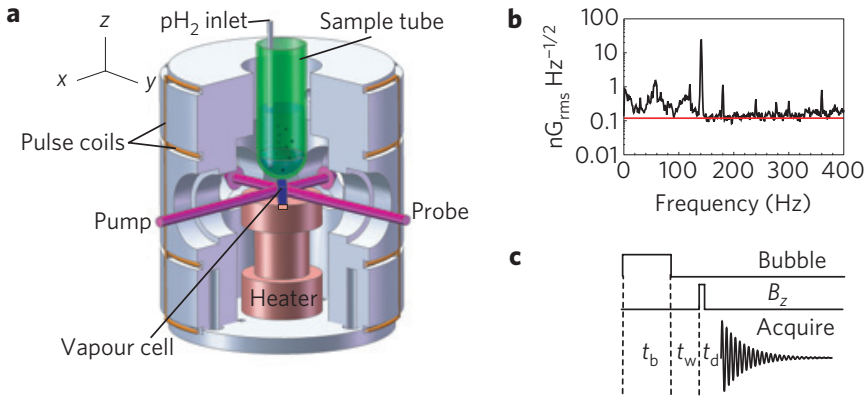

Figure 1 | Scheme for detecting parahydrogen-induced polarization at zero magnetic field. a, The experimental set-up. A microfabricated alkali vapour cell is mounted inside a set of coils used for applying magnetic-field pulses. The alkali vapour is optically pumped with a circularly polarized laser beam, resonant with the $\mathrm{D} 1$ transition of ${ }^{87} \mathrm{Rb}$. A linearly polarized laser beam, tuned about $100 \mathrm{GHz}$ off resonance, is used to probe the alkali spin precession. The magnetometer is primarily sensitive to magnetic fields in the vertical ( $z$ ) direction. A 7-mm-inner-diameter glass tube contains the sample, and a 1/32"-inner-diameter Teflon tube is used to bubble parahydrogen through the solution. A set of magnetic shields surrounding the magnetometer, not shown, isolates the magnetometer from external magnetic fields. $\mathbf{b}$, The magnetic-field noise spectrum of the magnetometer. Above $100 \mathrm{~Hz}$, the noise floor is about $0.15 \mathrm{nG} \mathrm{Hz}{ }^{-1 / 2}$. c, The experimental pulse sequence.

inset, with the blue carbon indicating the $\beta$ label and the green carbon indicating the $\alpha$ label. The spectrum of ethylbenzene- $\beta^{13} \mathrm{C}$ in Fig. 2a can be understood in terms of the discussion above, with multiplets at ${ }^{1} J_{\mathrm{HC}}$ and $2 \times{ }^{1} J_{\mathrm{HC}}$, and extra lines at low frequency. Here the superscript indicates the number of bonds separating the interacting pair, and ${ }^{1} J_{\mathrm{HC}}=126.2 \mathrm{~Hz}$ for ethylbenzene- $\beta^{13} \mathrm{C}$ (ref. 26). Isolated lines in the complex spectrum fit to complex Lorentzians with half-width at half-maximum of about $0.1 \mathrm{~Hz}$. It should be noted that this spectrum is similar to the correspondingly labelled ethanol- $\beta^{13} \mathrm{C}$ spectrum reported in ref. 4 , although careful inspection reveals small splittings of some lines due to long-range (at least four-bond) homonuclear couplings to protons on the benzene ring. The blue trace shows the result of a numerical simulation accounting for eight spins, including the six spins on the ethyl part of the molecule and the two nearest protons on the benzene ring. The simulation reproduces most of the features of the experimental spectrum quite well, including small splittings of several lines. More details of the numerical simulation can be found in Supplementary Information.

The zero-field PHIP spectrum of ethylbenzene- $\alpha^{13} \mathrm{C}$ shown in Fig. $2 \mathrm{~b}$ is qualitatively similar to the zero-field spectrum of ethanol- $\alpha^{13} \mathrm{C}$ (ref. 4), with a multiplet at roughly $3 / 2 \times{ }^{1} J_{H C}$ $\left({ }^{1} J_{\mathrm{HC}}=126.2 \mathrm{~Hz}\right.$, measured in house with a $300 \mathrm{MHz}$ spectrometer $)$ and features at low frequency. Many extra lines in the spectrum indicate that long-range couplings to the protons on the benzene ring are important. As the ethanol- $\beta^{13} \mathrm{C}$ spectrum does not show such complexity, the largest perturbation to the ethyl part of the molecule must be due to three-bond ${ }^{3} J_{\mathrm{HC}}$ couplings. The green trace shows the result of numerical simulation, consisting of the six spins on the ethyl part of the molecule and the two nearest protons on the benzene ring. Simulation again reproduces most of the features of the experimental spectrum, although careful inspection shows a number of extra splittings in the experimental spectrum, indicating that couplings to more remote spins on the benzene ring not included in the simulation are important. It is worth emphasizing that, despite the similarity of the one-bond heteronuclear J couplings, the spectra associated with different isotopomers show strikingly different features, which appear in different parts of the spectrum, facilitating easy assignment of isotopomers to their respective peaks. 


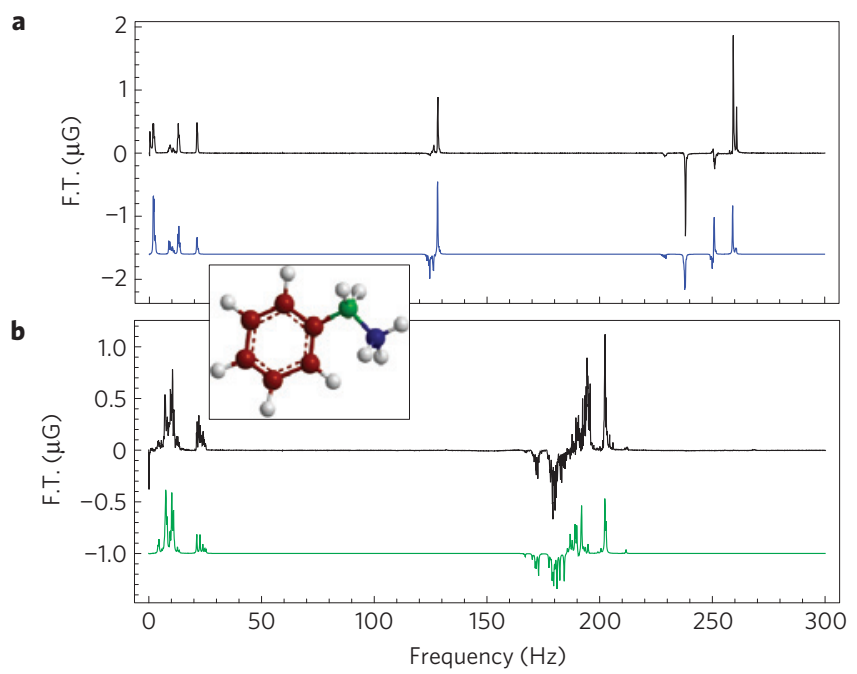

Figure 2 | Single-shot zero-field PHIP J-spectra (imaginary component). $\mathbf{a}, \mathbf{b}$, Ethylbenzene- $\beta^{13} \mathrm{C}(\mathbf{a})$ and ethylbenzene- $\alpha^{13} \mathrm{C}(\mathbf{b})$, polarized through addition of parahydrogen to labelled styrene. The inset shows the ethylbenzene molecule with the $\beta$ and $\alpha$ positions indicated by the blue and green carbons, respectively. The blue and green traces in $\mathbf{a}$ and $\mathbf{b}$, respectively, are the results of numerical simulations, described in the text.

The sensitivity of the magnetometer and the degree of parahydrogen-induced polarization are sufficient to detect $J$ spectra in compounds with ${ }^{13} \mathrm{C}$ in natural abundance. Figure 3 shows the zero-field PHIP spectrum of ethylbenzene with ${ }^{13} \mathrm{C}$ in natural abundance, obtained in just eight transients. The spectrum shown here is the sum of spectra associated with the $\alpha$ and $\beta$ isotopomers shown in Fig. 2, as well as isotopomers that carry ${ }^{13} \mathrm{C}$ in one of four non-equivalent positions on the benzene ring. The high-frequency parts of the spectrum arising from the $\alpha$ and $\beta$ isotopomers are highlighted in green and blue, respectively. The part of the signal arising from the benzene ring with a single ${ }^{13} \mathrm{C}$ is a multiplet centred about the one-bond coupling frequencies (typically about $156 \mathrm{~Hz}$ in aromatic systems), and also a multiplet in the low-frequency range. The high-frequency component is highlighted in red. Interestingly, spectra associated with the $\alpha$ or $\beta$ isotopomers do not overlap with spectra associated with isotopomers with ${ }^{13} \mathrm{C}$ on the benzene ring. It is also noteworthy that, if the hydrogenation is carried out in high field, large chemical-shift differences between protons on the benzene ring and the parahydrogen-derived protons would inhibit the transfer of polarization to the benzene ring.

To further illustrate the capabilities of zero-field PHIP as a method for chemical fingerprinting, spectra obtained from several different hydrogenation reactions are presented in Fig. 4: phenyl propyne (forming phenyl propene on hydrogenation) with a labelled ${ }^{13} \mathrm{CH}_{3}$ group (Fig. 4a), dimethyl acetylenedicarboxylate (dimethyl maleate) with ${ }^{13} \mathrm{C}$ in natural abundance (Fig. 4b) and 3-hexyne (hexene and hexane) with ${ }^{13} \mathrm{C}$ in natural abundance (Fig. 4c). These spectra are the result of averaging one, six and 32 transients respectively. The phenyl propene spectrum shows characteristics similar to the ethylbenzene- $\beta^{13} \mathrm{C}$ spectrum, although the phase and splitting pattern is clearly different because neither of the parahydrogen-derived protons are part of the labelled group. The dimethyl maleate spectrum shown in Fig. $4 \mathrm{~b}$ is the superposition of two different ${ }^{13} \mathrm{C}$ isotopomers, and can approximately be understood as follows. For a three-spin system, where one of the parahydrogen-derived spins has a strong coupling to a ${ }^{13} \mathrm{C}$ nucleus, we can show that the spectrum consists of two lines centred around the strong-coupling frequency, and an extra low-frequency peak. The antiphase lines centred about $165 \mathrm{~Hz}$ in Fig. $4 \mathrm{~b}$ correspond to the isotopomer where the ${ }^{13} \mathrm{C}$

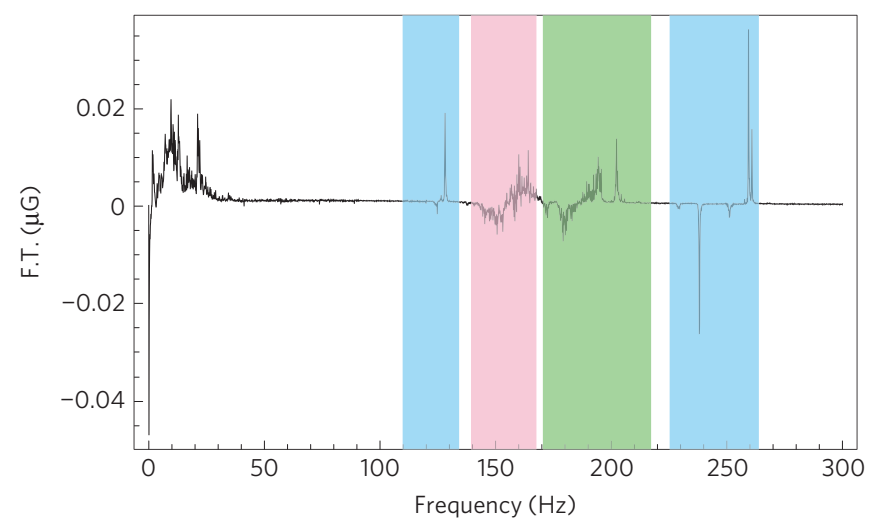

Figure 3 | Zero-field $J$ spectrum (imaginary component) of ethylbenzene, produced through parahydrogenation of styrene with ${ }^{13} \mathrm{C}$ in natural abundance. These data result from averaging eight transients following a pulse of magnetic field in the $z$ direction with $\eta \approx \pi / 2$. The high-frequency components of the signals arising from the $\alpha$ and $\beta$ isotopomers are easily recognizable from the spectra shown in Fig. 2, and are highlighted by the green and blue bands, respectively. The signal in the neighbourhood of $156 \mathrm{~Hz}$ is due to isotopomers with ${ }^{13} \mathrm{C}$ on the benzene ring, and is highlighted in red.

is directly bonded to one of the parahydrogen-derived spins, and is accompanied by a contribution at low frequency. The other three-spin isotopomer, where the strongest coupling to the ${ }^{13} \mathrm{C}$ nucleus is through two bonds, nominally gives rise to three lines at low frequency. There are some residual splittings in the low-frequency part of the spectrum, which will be the subject of future investigation. The spectrum obtained in the hexyne reaction in Fig. $4 \mathrm{c}$ is the sum of three different ${ }^{13} \mathrm{C}$ isotopomers. For labelled ${ }^{13} \mathrm{CH}_{3}$ groups, signal arises at ${ }^{1} J_{\mathrm{HC}}$ and $2 \times{ }^{1} J_{\mathrm{HC}}$, where ${ }^{1} J_{\mathrm{HC}} \approx 125 \mathrm{~Hz}$. For labelled ${ }^{13} \mathrm{CH}_{2}$ groups, the contribution to the signal is centred about $3 J / 2$, producing signal in the range of $170-200 \mathrm{~Hz}$. Long-range couplings to other spins yield extra splitting. A more detailed discussion of these spectra and rules for assigning transitions and understanding zero-field PHIP spectra will be presented in a forthcoming publication.

\section{Outlook}

Finally, we make several observations. (1) Here we operate in zero magnetic field. Working in small but finite fields on the order of $1 \mathrm{mG}$ may yield further information regarding molecular structure, albeit at the expense of extra spectral complexity ${ }^{27}$. (2) A common objection to low- and zero-field NMR is that spectra become complex as the number of spins increases, as exemplified by comparison of the ethanol- $\alpha^{13} \mathrm{C}$ spectrum reported in ref. 4 and the ethylbenzene- $\alpha^{13} \mathrm{C}$ obtained here. The increasing complexity of spectra with spin system size is a feature that is also encountered in standard high-field NMR, and has been successfully addressed by application of multiple-pulse sequences and multidimensional spectroscopy. The theory of multiple-pulse sequences for zero-field NMR was worked out some time ago ${ }^{28}$, and presumably many of the techniques developed for high field could be adapted to zero field. (3) We achieve linewidths of about $0.1 \mathrm{~Hz}$. For ${ }^{13} \mathrm{C}-\mathrm{H} J$-coupled systems, the dispersion in signal is about $300 \mathrm{~Hz}$, so roughly 1,500 lines can fit in a spectrum without overlapping. This is similar to what may be achieved in a $400 \mathrm{MHz}$ spectrometer if we assume proton chemical shifts ranging over $6 \mathrm{ppm}$ and proton linewidths of about $0.5 \mathrm{~Hz}$. (4) The sensitivity of the magnetometer used in this work was about $0.15 \mathrm{nG} \mathrm{Hz}^{-1 / 2}$ using a vapour cell with a volume of $10 \mathrm{~mm}^{3}$. Sensitivities about two orders of magnitude better have been achieved in larger vapour cells ${ }^{29}$, which will enable measurements on larger samples with much lower concentration. 


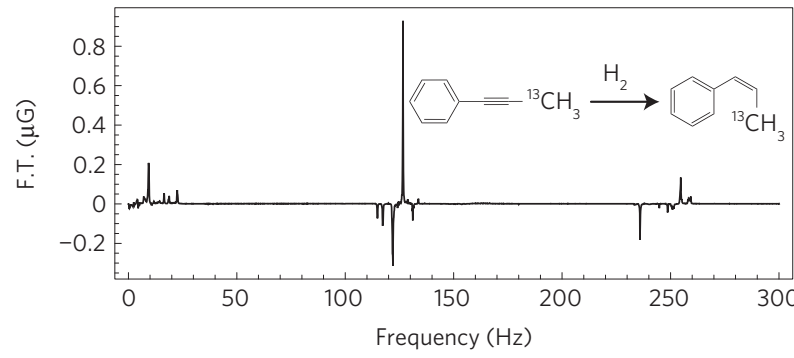

b
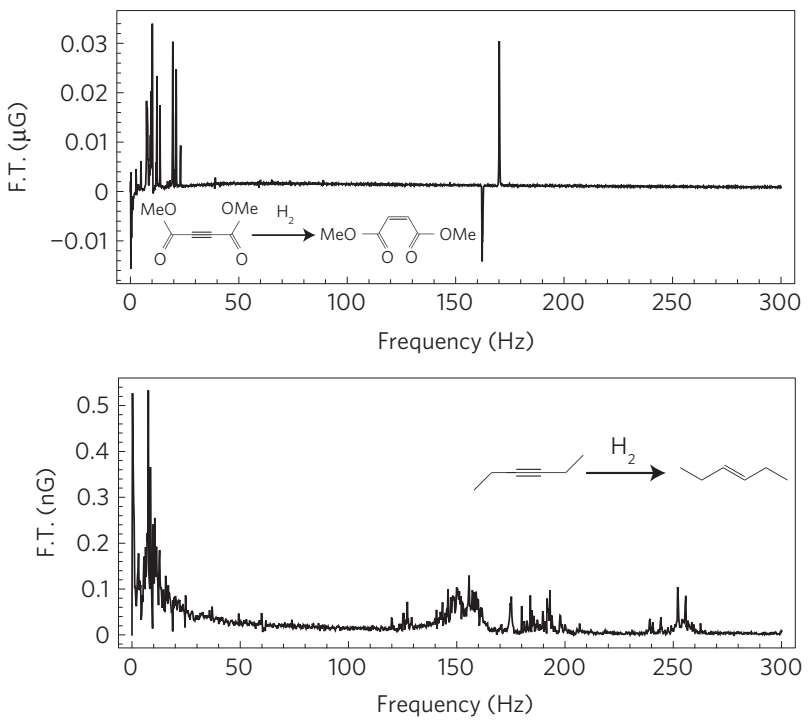

Figure 4 | Zero-field PHIP spectra for several compounds. a, Parahydrogen is added to 1-phenyl-1-propyne, labelled with ${ }^{13} \mathrm{C}$ in the $\mathrm{CH}_{3}$ group. b, Parahydrogen is added to acetylene dimethylcarboxylate with ${ }^{13} \mathrm{C}$ in natural abundance. $\mathbf{c}$, Parahydrogen is added to 3 -hexyne with ${ }^{13} \mathrm{C}$ in natural abundance. In $\mathbf{a}$ and $\mathbf{b}$ the imaginary component is presented; in $\mathbf{c}$ the magnitude is presented.

In conclusion, we have demonstrated NMR without the use of any magnets by using parahydrogen-induced polarization and a high-sensitivity atomic magnetometer with a microfabricated vapour cell. The mechanism by which the symmetry of the singlet states is broken in zero field relies only on the presence of heteronuclear $J$ coupling and not chemical shifts, in contrast to many experiments carried out in high field. Hydrogen-carbon $J$ couplings through at least three bonds and hydrogen-hydrogen couplings through four bonds are observed. We also observe that polarization is naturally transferred through several bonds to remote parts of the molecule. This can be contrasted with in situ hydrogenation in high field, where chemical shifts larger than $J$ couplings prevent efficient polarization transfer without the use of auxiliary radiofrequency pulses. Sensitivity is sufficient to carry out $J$ spectroscopy on samples with ${ }^{13} \mathrm{C}$ in natural abundance with very little signal averaging. The resulting spectra, despite exhibiting a large number of lines, can easily be divided into different parts, which can directly be assigned to different isotopomers of the molecule at hand. Although our technique may seem to be limited to molecules to which hydrogen can be added, recent advances using iridium complex catalysts enable polarization of molecules without hydrogenation ${ }^{18,19}$, significantly expanding the scope of applicability of zero-field PHIP. As the development of zero-field NMR is still at an early stage it is not possible to fully gauge its competitiveness with highfield NMR or portable lower-resolution versions thereof, but it clearly has potential to become a low-cost, portable method for chemical analysis.

\section{Methods}

Experimental set-up. The zero-field spectrometer is similar to that of ref. 4 and is shown schematically in Fig. 1a. An atomic magnetometer, consisting of a Rb vapour cell and two lasers for optical pumping and probing, operates in the spin-exchange relaxation-free ${ }^{8}$ regime. The cell is placed inside a set of magnetic shields (not shown), and residual magnetic fields are zeroed to within $\approx 1 \mu \mathrm{G}$. The vapour cell has dimensions $5 \mathrm{~mm} \times 2 \mathrm{~mm} \times 1 \mathrm{~mm}$, contains ${ }^{87} \mathrm{Rb}$ and 1,300 torr of $\mathrm{N}_{2}$ buffer gas and was microfabricated using lithographic patterning and etching techniques. The cell is heated to $210^{\circ} \mathrm{C}$ through an electric heating element wound around an aluminium nitride spool. The sensitivity of the magnetometer is about $0.15 \mathrm{nG} \mathrm{Hz}^{-1 / 2}$ above $120 \mathrm{~Hz}$, and the bandwidth is in excess of $400 \mathrm{~Hz}$. A set of coils can be used to apply sharp, $\approx 1 \mathrm{G}$ d.c. pulses in arbitrary directions to excite NMR coherences, and a separate set of coils (not shown) controls the ambient magnetic field inside the shields. Mixtures of catalyst, solvent and substrate could be brought into proximity with the atomic magnetometer through a glass sample tube. The sample was maintained at $80^{\circ} \mathrm{C}$ by flowing air through a jacket surrounding the glass tube. In experiments at lower temperature (not presented here), we found that there was some non-uniform broadening of spectra, presumably due to the presence of catalyst in solid form. Parahydrogen was bubbled through the solution through a 0.8 -mm-inner-diameter tube for several seconds at a pressure of about 70 PSI and flow rate of about 120 s.c.c.m. Bubbling was halted before application of excitation pulses and signal acquisition. Data were acquired with a sampling rate of $2 \mathrm{kS} \mathrm{s}^{-1}$. In acquiring the spectrum of styrene with natural-abundance ${ }^{13} \mathrm{C}$, the phase of the excitation pulses was cycled with respect to that of the $60 \mathrm{~Hz}$ line frequency to reduce the line noise and its harmonics.

Production of parahydrogen. Parahydrogen was produced at $29 \mathrm{~K}$ by flowing hydrogen gas through a bed of iron oxide catalyst in a set-up similar to that described in ref. 30 and then stored in an aluminium canister at room temperature and initial pressure of 150 PSI. Conversion of hydrogen to parahydrogen was about $95 \%$, and storage lifetime was in excess of one week.

Sample preparation. Isotopically labelled styrene was obtained from Cambridge Isotope Labs. Natural-abundance styrene and Wilkinson's catalyst ${ }^{31}$ were obtained from Sigma-Aldrich. Styrene hydrogenations were carried out with $300 \mu \mathrm{l}$ styrene and $4 \mathrm{mG}$ Wilkinson's catalyst, tris(triphenylphosphine)rhodium(I) chloride (CAS no 14694-95-2). The 1-phenyl-propyne and dimethyl acetylenedicarboxylate reactions were carried out with $100 \mu \mathrm{l}$ substrate in $300 \mu \mathrm{l}$ tetrahydrofuran, catalysed by 1,4-bis(diphenylphosphino)butane](1,5cyclooctadiene)rhodium(I)tetrafluoroborate, (CAS no 79255-71-3). The hexyne reaction was carried out in a solution of $50 \%$ tetrahydrofuran of $5 \mathrm{ml}$ in total and $30 \mathrm{mG}$ Wilkinson's catalyst. Most of this volume does not contribute to signal because it is far from the magnetometer.

Received 14 December 2010; accepted 22 March 2011; published online 1 May 2011

\section{References}

1. Ernst, R. R., Bodenhausen, G. \& Wokaun, A. Principles of Nuclear Magnetic Resonance in One and Two Dimensions (Oxford Univ. Press, 1987).

2. Slichter, C. P. Principles of Magnetic Resonance 3rd edn (Springer, 1990).

3. Appelt, S., Häsing, F. W., Kühn, H., Perlo, J. \& Blümich, B. Mobile high resolution xenon nuclear magnetic resonance spectroscopy in the Earth's magnetic field. Phys. Rev. Lett. 94, 197601 (2005).

4. McDermott, R. et al. Liquid-state NMR and scalar couplings in microtesla magnetic fields. Science 295, 2247-2249 (2002).

5. Ledbetter, M. P. et al. Optical detection of NMR $J$-spectra at zero magnetic field. J. Magn. Res. 199, 25-29 (2009).

6. Appelt, S., Kühn, H., Häsing, F. W. \& Blümich, B. Chemical analysis by ultrahigh-resolution nuclear magnetic resonance in the Earths magnetic field. Nature Phys. 2, 105-109 (2006).

7. Budker, D. \& Romalis, M. V. Optical magnetometry. Nature Phys. 3, 227-234 (2007).

8. Kominis, I. K., Kornack, T. W., Allred, J. C. \& Romalis, M. V. A sub-femtoTesla multichannel atomic magnetometer. Nature 422, 596-599 (2003).

9. Greenberg, Y. S. Application of superconducting quantum interference devices to nuclear magnetic resonance. Rev. Mod. Phys. 70, 175-222 (1998).

10. Ledbetter, M. P. et al. Zero-field remote detection of NMR with a microfabricated atomic magnetometer. Proc. Natl Acad. Sci. USA 105, 2286-2290 (2008).

11. Savukov, I. M. \& Romalis, M. V. NMR detection with an atomic magnetometer. Phys. Rev. Lett. 94, 123001 (2005).

12. Xu, S. J. et al. Magnetic resonance imaging with an optical atomic magnetometer. Proc. Natl Acad. Sci. USA 103, 12668-12671 (2006).

13. Savukov, I. M. et al. MRI with an atomic magnetometer suitable for practical imaging applications. J. Magn. Res. 199, 188-191 (2009).

14. Bowers, C. R. \& Weitekamp, D. P. Transformation of symmetrization order to nuclear-spin magnetization by chemical-reaction and nuclear-magnetic-resonance. Phys. Rev. Lett. 57, 2645-2648 (1986). 
15. Natterer, J. \& Bargon, J. Parahydrogen induced polarization. Prog. Nucl. Magn. Reson. Spectrosc. 31, 293-315 (1997).

16. Bowers, C. R. in Encylopedia of Nuclear Magnetic Resonance Vol. 9 (eds Grant, D. M. \& Harris, R. K.) 750-770 (2002).

17. Canet, D. et al. Para-hydrogen enrichment and hyperpolarization. Concepts Magn. Reson. Part A 28A, 321-330 (2006).

18. Adams, R.W. et al. Reversible interactions with para-hydrogen enhance NMR sensitivity by polarization transfer. Science 323, 1708-1711 (2009).

19. Atkinson, K. D. et al. Spontaneous transfer of parahydrogen induced spin order to pyridine at low magnetic field. J. Am. Chem. Soc. 131, 13362-13368 (2009).

20. Chapovsky, P. L. et al. Separation and conversion of nuclear spin isomers of ethylene. Chem. Phys. Lett. 322, 424-428 (2000).

21. Sun, Z-D., Takagi, K. \& Matsushima, F. Separation and conversion of four nuclear spin isomers of ethylene. Science 310, 1938-1941 (2005).

22. Aime, S., Gobetto, R., Reineri, F. \& Canet, D. Polarization transfer from parahydrogen to heteronuclei: The effect of H/D substitution. The case of the $\mathrm{AA}^{\prime} \mathrm{X}$ and $\mathrm{A}_{2} \mathrm{~A}_{2}^{\prime} \mathrm{X}$ spin systems. J. Magn. Res. 178, 184-192 (2006).

23. Carravetta, M., Johannessen, O. G. \& Levitt, M. H. Beyond the $T_{1}$ limit: Singlet nuclear spin states in low magnetic fields. Phys. Rev. Lett. 92, 153001 (2004).

24. Pileio, G., Carravetta, M. \& Levitt, M. H. Extremely low-frequency spectroscopy in low-field nuclear magnetic resonance. Phys. Rev. Lett. 103, 083002 (2009).

25. Zax, D. B., Bielecki, A., Zilm, K. W. \& Pines, A. Heteronuclear zero-field NMR. Chem. Phys. Lett. 106, 550-553 (1984).

26. Schaefer, T., Chan, W. K., Sebastian, R., Schurko, R. \& Hruxka, F. E Concerning the internal rotational barrier and the experimental and theoretical ${ }^{n} \mathrm{~J}\left({ }^{13} \mathrm{C},{ }^{13} \mathrm{C}\right)$ and ${ }^{n} \mathrm{~J}\left({ }^{1} \mathrm{H},{ }^{13} \mathrm{C}\right)$ in ethylbenzene- $\beta{ }^{13} \mathrm{C}$. Can. J. Chem. 72, 1972-1977 (1994).

27. Appelt, S. et al. Paths from weak to strong coupling in NMR. Phys. Rev. A 81, 023420 (2010).

28. Lee, C. J., Suter, D. \& Pines, A. Theory of multiple-pulse NMR at low and zero fields. J. Magn. Res. 75, 110-124 (1987).
29. Dang, H. B., Maloof, A. C. \& Romalis, M. V. Ultrahigh sensitivity magnetic field and magnetization measurements with an atomic magnetometer. Appl. Phys. Lett. 97, 151110 (2010).

30. Koptyug, I. V. et al. Para-hydrogen induced polarization in heterogeneous hydrogenation reactions. J. Am. Chem. Soc. 129, 5580-5586 (2007).

31. Osborn, J. A., Jardine, F. H., Young, J. F. \& Wilkinson, G. The preparation and properties of tris(triphenylphosphine)halogenorhodium(I) and some reactions thereof including catalytic homogeneous hydrogenation of olefins and acetylenes and their derivatives. J. Chem. Soc. A 1711-1732 (1966).

\section{Acknowledgements}

Research was supported by the US Department of Energy, Office of Basic Energy Sciences, Division of Materials Sciences and Engineering under Contract no DE-AC02-05CH11231 (T.T., P.G., G.K. and A.P.), by the National Science Foundation under award noCHE-0957655 (D.B. and M.P.L.) and by the National Institute of Standards and Technology (S.K. and J.K.). We acknowledge discussions with M. Levitt and magnetometer-cell fabrication help from S. Schima.

\section{Author contributions}

T.T. designed research, carried out experiments and wrote the paper. P.G. contributed to construction of the experiment. G.K. carried out experiments and theoretical analysis. S.K. and J.K. provided the microfabricated vapour cell. M.P.L. built the experiment, designed research, carried out experiments, simulations and theoretical analysis and wrote the paper. D.B. designed research and wrote the paper. A.P. designed research and wrote the paper.

\section{Additional information}

The authors declare no competing financial interests. Supplementary information accompanies this paper on www.nature.com/naturephysics. Reprints and permissions information is available online at http://www.nature.com/reprints. Correspondence and requests for materials should be addressed to A.P. 